\title{
Development and validation of a noncontact spectroscopic device for hemoglobin estimation at point-of- care
}

Probir Kumar Sarkar

Sanchari Pal

Nabarun Polley

Rajarshi Aich

Aniruddha Adhikari

Animesh Halder

Subhananda Chakrabarti

Prantar Chakrabarti

Samir Kumar Pal 


\title{
Development and validation of a noncontact spectroscopic device for hemoglobin estimation at point-of-care
}

\author{
Probir Kumar Sarkar, ${ }^{a}$ Sanchari Pal, ${ }^{b}$ Nabarun Polley, ${ }^{a}$ Rajarshi Aich, ${ }^{c}$ Aniruddha Adhikari, ${ }^{a}$ Animesh Halder, ${ }^{a}$ \\ Subhananda Chakrabarti, ${ }^{d}$ Prantar Chakrabarti, ${ }^{b}$ and Samir Kumar Pal ${ }^{a, *}$ \\ as. N. Bose National Centre for Basic Sciences, Department of Chemical, Biological, and Macromolecular Sciences, Salt Lake, Kolkata, India \\ ${ }^{b}$ Nil Ratan Sircar Medical College and Hospital, Department of Clinical Haematology, Sealdah, Kolkata, West Bengal, India \\ 'Medical College and Hospital, Department of Cardiology, Central Avenue, Kolkata, West Bengal, India \\ dIndian Institute of Technology Bombay, Department of Electrical Engineering, Powai, Mumbai, Maharashtra, India
}

\begin{abstract}
Anemia severely and adversely affects human health and socioeconomic development. Measuring hemoglobin with the minimal involvement of human and financial resources has always been challenging. We describe a translational spectroscopic technique for noncontact hemoglobin measurement at low-resource point-of-care settings in human subjects, independent of their skin color, age, and sex, by measuring the optical spectrum of the blood flowing in the vascular bed of the bulbar conjunctiva. We developed software on the LabVIEW platform for automatic data acquisition and interpretation by nonexperts. The device is calibrated by comparing the differential absorbance of light of wavelength 576 and $600 \mathrm{~nm}$ with the clinical hemoglobin level of the subject. Our proposed method is consistent with the results obtained using the current gold standard, the automated hematology analyzer. The proposed noncontact optical device for hemoglobin estimation is highly efficient, inexpensive, feasible, and extremely useful in low-resource point-of-care settings. The device output correlates with the different degrees of anemia with absolute and trending accuracy similar to those of widely used invasive methods. Moreover, the device can instantaneously transmit the generated report to a medical expert through e-mail, text messaging, or mobile apps. ๑ 2017 Society of Photo-Optical Instrumentation Engineers (SPIE) [DOI: 10 1117/1.JBO.22.5.055006]
\end{abstract}

Keywords: hemoglobin estimation; noncontact; optical device; anemia screening; point-of-care.

Paper 170037RR received Jan. 16, 2017; accepted for publication Apr. 26, 2017; published online May 16, 2017.

\section{Introduction}

Anemia is one of the most common global health problems affecting both developed and developing countries; it has farreaching and severe adverse effects on human health and strongly affects socioeconomic development. ${ }^{1}$ Approximately two billion people - nearly $30 \%$ of the world population-are estimated to be currently anemic, ${ }^{2-4}$ and women of reproductive age, pregnant or breastfeeding women, and children are the most vulnerable populations. ${ }^{5-7}$ The 2015 World Health Organization (WHO) fact sheets on the global statistics of anemia show that roughly 273 million (43\%) children, 32.4 million (38\%) pregnant women, 496 million (29\%) nonpregnant women, and $29 \%$ of all women of reproductive age are anemic. ${ }^{8,9}$ Therefore, anemia detection and control activities should be an integral part of healthcare services, particularly because an early diagnosis of anemia in the population is a proven means of health promotion.

Hemoglobin measurement is usually dependent on the services of a well-equipped clinical laboratory. The current gold standard, automated hematology analyzers, uses blood samples for precisely estimating hemoglobin concentration in a laboratory setting. ${ }^{7}$ Although this method is effective, it has several drawbacks. Numerous other methods, such as HemoCue, hemoglobin color scale, copper sulfate method, are available, but all

*Address all correspondence to: Samir Kumar Pal, E-mail: skpal@ bose.res.in of these methods require a high level of technical skills to interpret and at least one drop of blood. ${ }^{10}$ Invasive blood sampling is stressful and painful for the patients and results in blood loss, which may induce anemia in infants and infection at the sampling site. ${ }^{11}$ In addition, the accuracy of the results is heavily dependent on the skills of the operators. ${ }^{12}$ Moreover, hematology analyzers are expensive, ${ }^{10}$ and the cost per sample is significantly high compared with older manual techniques.

To date, the lack of a portable, easily operable, inexpensive, and accurate device has hindered the widespread adaptation of anemia screening in public health programs. ${ }^{13}$ Most anemic women live in low-resource areas, where cost-effective and accurate diagnosis of anemia is unavailable. These factors have encouraged researchers and manufacturers to develop an economic and accurate noninvasive device for anemia detection in point-of-care settings; such a device would increase the a ccessibility of anemia screening and increase early diagnosis and treatment. ${ }^{14}$ Some methods for point-of-care noninvasive hemoglobin measurements, such as Pronto-7TM and Pulse CO-Oximeter developed by Masimo Corporation (Irvine, California), NBM-200 developed by OrSense (Israel), and spectrophotometry, have been described in the literature. ${ }^{14-18}$ Most of these devices use a finger probe, and the technology is analogous to noninvasive pulse oximetry, which measures the total hemoglobin level within a few minutes. However, 
although these devices address many problems encountered in older noninvasive devices, they have their own limitations, ${ }^{14}$ such as low precision and accuracy. ${ }^{19}$ The accuracy and sensitivity of all noninvasive devices vary across races (specifically, skin color) because of the variation of melanin concentration in skin tissues. ${ }^{17,20}$ Moreover, they are not highly efficient and reliable as they frequently produce erroneous results because of the limitations associated with direct access to the blood or blood vessels and change in tissue morphology, shape, and blood content in the targeted area. ${ }^{17}$

To overcome the aforementioned limitations and to realize several crucial functions missing in the current noninvasive devices, we applied a spectroscopic method and addressed many unavoidable requirements that are missing in the previous noninvasive devices. In this paper, we present a noninvasive, noncontact, and portable device for hemoglobin estimation at point-of-care in human subjects. Our innovation is based on the measurement of the spectroscopic signal emanating from the vascular bed of the bulbar conjunctiva. ${ }^{21}$ As the conjunctiva in all humans is transparent and has white sclera as background, ${ }^{22}$ the accuracy and sensitivity of the proposed device are independent of the skin color of the subjects. The easy access to the conjunctiva and its high vascular visibility ensures that the proposed device has high accuracy and sensitivity. The other distinguishing features of this device include the following: (1) direct measurements of the hemoglobin level with extremely high precision and accuracy and without interference from pathological conditions, (2) the noninvasive and noncontact nature of the device, which is essential for use in neonates and virus-infected maternal subjects, (3) online monitoring and compatibility with mobile-phone platforms for data transceiving, which facilitates the quick development of treatment plans, (4) almost zero maintenance costs, and (5) operable by minimally trained or nearly untrained healthcare workers. Moreover, the proposed device can efficiently estimate hemoglobin levels with high precision and accuracy and without requiring any blood samples or chemical reagents at point-ofcare. Furthermore, the generated report can be instantaneously transmitted to a medical expert through e-mail, text messaging, or mobile apps. Thus, the proposed device has high potential for further development and commercialization.

\section{Methods}

\subsection{Experimental Setup}

Oxyhemoglobin has a unique characteristic optical spectrum over the visible light range, with absorption peaks at $\sim 414$, 542 , and $576 \mathrm{~nm} \cdot{ }^{23,24}$ Figure 1(a) presents an optical fiber and spectroscopy-based absorbance setup for collecting and monitoring the diffused reflectance spectral response of the blood flowing in the vascular bed of the bulbar conjunctiva of human subjects (patent 466/KOL/2009). A spectrograph (STSVIS) with wavelength resolution of $0.47 \mathrm{~nm}$ and a white lightemitting diode (LED) source [LS-450, with the preinstalled blue LED replaced with a white LED (196-00000-WHT)] with wavelength range of 430 to $700 \mathrm{~nm}$ (Ocean Optics, Florida) were used in our study. A lab-grade optical fiber probe (Fibertype UV-VIS; Ocean Optics) was used for transmitting and collecting the light to and from the vascular bed of the bulbar conjunctiva. The light from the light source is carried by the six surrounding fibers of the probe (called excitation fibers) and is incident on the conjunctiva, and the single fiber in the middle
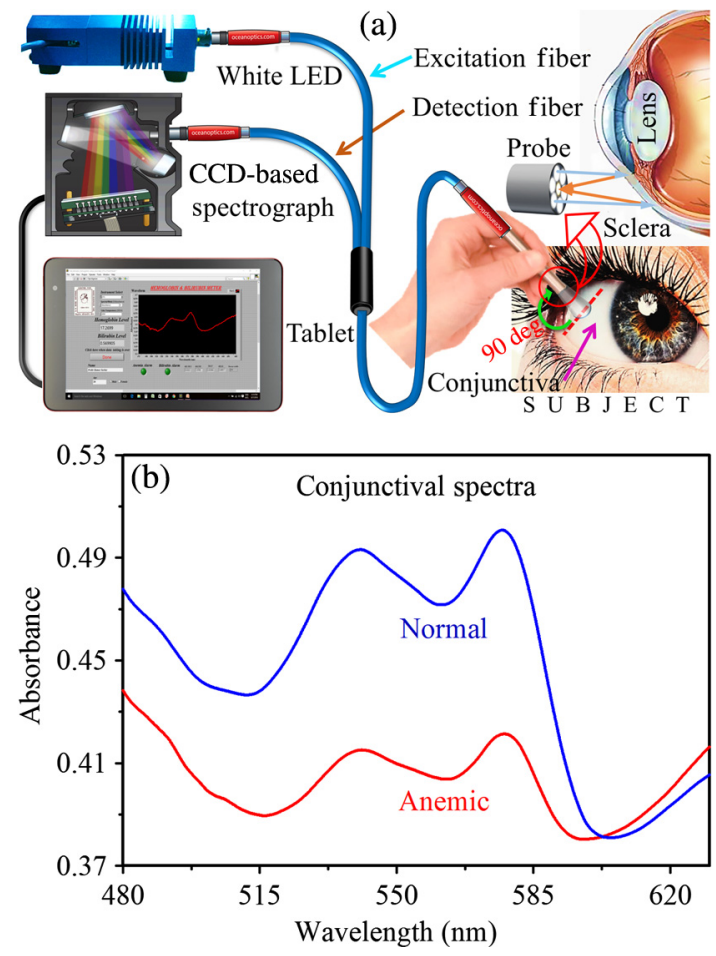

Fig. 1 (a) Schematic representation of the proposed device. The light from the source is transmitted through the six-channel excitation fiber and incident on the conjunctiva of the subject. The diffused light from the conjunctiva is collected using the single-channel detection fiber and transmitted to the spectrograph. The corresponding spectral response is processed and generated in the tablet. (b) Comparative spectral response of the conjunctiva of a normal volunteer and anemic patient.

of the probe (called the collection/detection fiber) collects the diffused light and transmits it back to the spectrograph [Fig. 1(a)]. The collected spectral response generated in the spectrograph is then transferred to a tablet computer through a Universal Serial Bus (USB) connection, where it is processed using custom-developed LabVIEW software. Figure 1(b) presents the collected comparative spectral response of a normal volunteer with hemoglobin level $16.6 \mathrm{~g} / \mathrm{dL}$ and an anemic patient with hemoglobin level $8.7 \mathrm{~g} / \mathrm{dL}$.

\subsection{Software Design}

For automatic data acquisition (DAQ) and visualization of the acquired spectra, the supporting software was designed on a LabVIEW platform. First, the collected spectral response as generated in the spectrograph is transferred to a tablet computer through a USB connection, where it is processed and displayed by a custom-designed LabVIEW software. The measured spectrum from the conjunctiva of the patient is displayed online and can be used to analyze the medical condition of the patient. Finally, the calculated hemoglobin level of the patient is displayed along with suggested medical attention on the screen of the tablet computer. Figure 2(a) illustrates the sequential program flow diagram of the developed software. The instrument is first initialized to its power-on status. Then, the software creates a folder for a given subject name to store the data and hemoglobin level of the subject. To achieve sufficient signal-tonoise $(\mathrm{S} / \mathrm{N})$ ratio in the collected data (spectrum), we set $2 \mathrm{~s}$ as the integration time throughout this study. The integration 

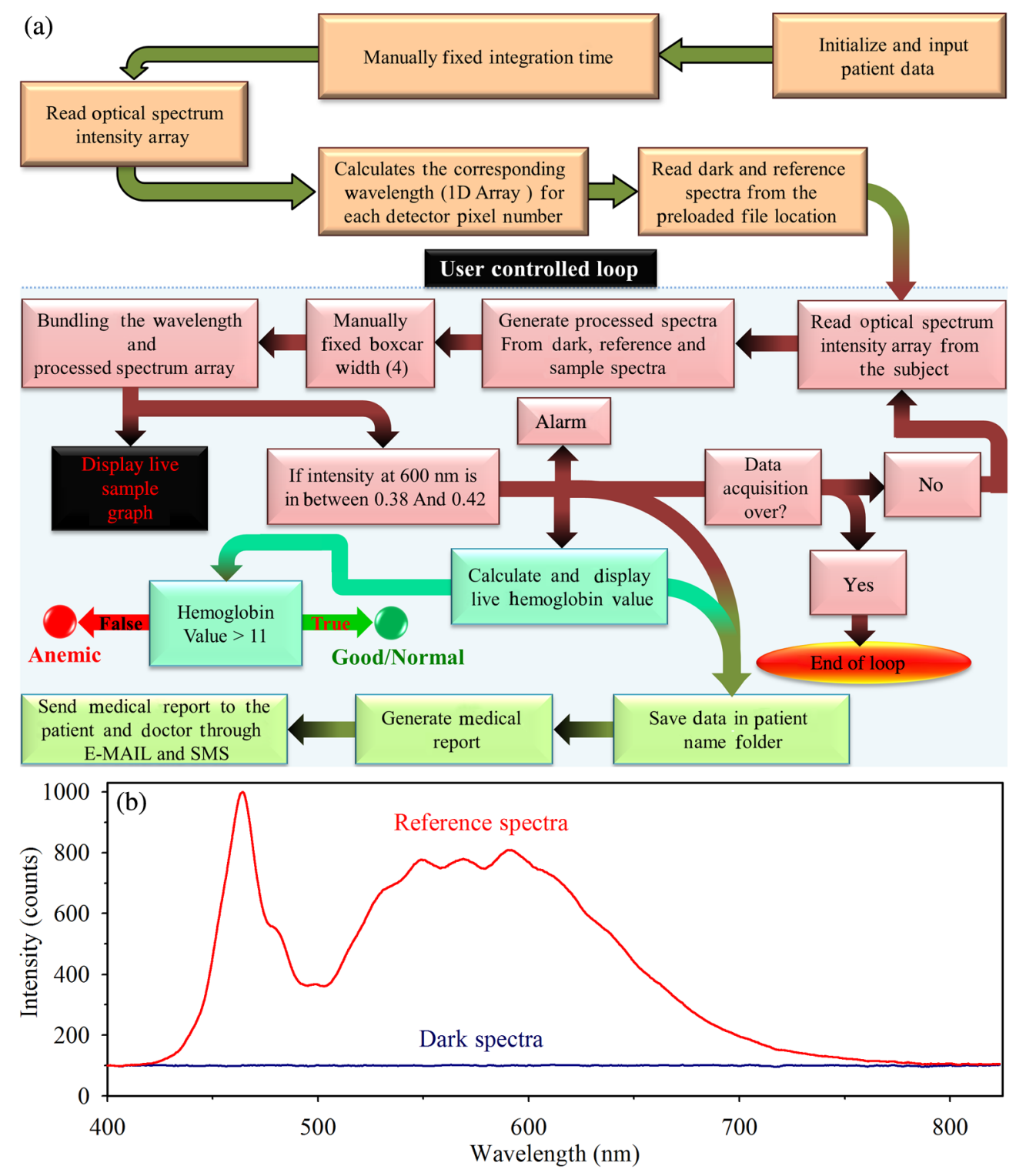

Fig. 2 (a) Sequential program flow diagram of the developed software designed on a LabVIEW platform for noncontact online monitoring of hemoglobin level in humans. (b) Typical reference and dark spectra.

time was determined at the time of reference spectrum collection. The reference spectrum is collected using a standard scatterer (WS-1 Reflectance Standards, Ocean Optics) with a spot size of diameter $\sim 0.7 \mathrm{~cm}$; the perpendicular distance between the reference surface and the probe tip was maintained at $\sim 1.5 \mathrm{~cm}$ by adjusting the integration time $(2 \mathrm{~s})$. The dark spectrum is acquired in the presence of ambient light by turning off the source LED. The length of the optical spectrum intensity array is fixed on the basis of the pixel number of the detector. Accordingly the corresponding wavelength array (one-dimensional array) is determined. The preacquired dark spectrum and reference spectrum, which were acquired each day before starting the data collection, are then read from the preloaded file location for spectrum processing and baseline correction. The software is then ready to read the optical spectrum intensity array from the subject and generate the processed spectrum array from the dark, reference, and sample spectra by using the below equation.
Processed spectrum

$$
=-\log _{10}\left[\frac{\text { Sample spectrum }- \text { Dark spectrum }}{\text { Reference spectrum }- \text { Dark spectrum }}\right] \text {. }
$$

Figure 2(b) shows the typical dark and reference spectra. The boxcar width $(=4)$ is then fixed to generate a smooth online graph by bundling the wavelength and processed spectrum array; this processing removes noise by averaging the values of the adjacent pixels and therefore improves the $\mathrm{S} / \mathrm{N}$ ratio at the expense of optical resolution. The online graph and data are automatically saved in the folder location with a sound alarm only when the absorbance intensity of the spectrum at $600 \mathrm{~nm}$ is between 0.38 and 0.42 . Setting this narrow range of absorption ensures the collection of spectral data from the spot size of diameter $\sim 0.7 \mathrm{~cm}$ on the conjunctiva. This spot size is the same as that used to acquire the reference spectrum. Subsequently, the software asks the user to either end the DAQ 
by ending the loop or to repeat the measurement on the same subject.

Using this developed software, we collected 302 participants' data to calibrate our instrument for hemoglobin measurements (Sec. 3.1). The calibration equation was incorporated into the software [Fig. 2(a)] for calculating the hemoglobin level from the acquired spectrum. Now the software can also display and save the calculated hemoglobin value following the earlier data saving conditions. The hemoglobin level of the patient is calculated using the differential absorption of light of wavelength 576 and $600 \mathrm{~nm}$. On the basis of the calculated hemoglobin level, the online display indicator classifies the data of the patient as being within or outside the anemic region. Finally, the acquired absorbance spectrum and the displayed hemoglobin level are stored in a folder (labeled with the name of the patient), and a comprehensive medical report is instantaneously generated and sent to the doctor and patient through e-mail and text messaging for offline use. We have ensured that the user interface of the software is appropriate for use by personnel with zero or minimal medical and instrumentation knowledge.

\subsection{Data Collection}

The study included a total of 493 patients (including 276 males, 74 of whom were children) with a variety of age groups ranging from 4 to 76 years [mean age \pm standard deviation $(\mathrm{SD})=$ $27 \pm 13$ years]. All data were collected using our device in the blood collection room of the outpatient department of Nil Ratan Sircar Medical College and Hospital, Kolkata, following the standard ethical guideline approved by the local medical ethics committee (No/NMC/443, dated January 25, 2016). A duly signed consent form was obtained from the patients or their legal guardians stating their acceptance, involvement, and full understanding of the study. All ethical guidelines, including participant confidentiality and legitimacy, were followed.

The study was conducted in two stages. In the first stage, the device was calibrated, and in the second, the accuracy of the software-driven device was measured and compared with that of the gold standard (i.e., the standard biochemical method). Because the study was conducted over a period of 120 days, the dark and reference spectra were acquired each day before starting the data collection to avoid the potential effects of variation in ambient light. We also reacquired both spectra in case of a change in the measurement location. Before acquisition of the dark and reference spectra, the light source was run for $5 \mathrm{~min}$ (according to manufacturer instructions, $<1.0 \%$ variation after 2 min of warm up). After completing the instrumental setup and preliminary legal and ethical formalities, the patient was instructed to sit on a chair and look in the direction opposite to that of the probe without head or eye movement. Data were acquired from the vascular bed of the bulbar conjunctiva by placing the probe close to ( $\sim 1.5 \mathrm{~cm}$ away from $)$ the patient's eye, approximately perpendicular to the surface of the conjunctiva [Fig. 1(a)]. Because the exposure of light to the conjunctiva is low ( $\sim 5 \mathrm{~s})$, no intervention (manual holding) was needed for most of the subjects. For more accurate measurement, we requested the subjects not to blink for a longer time (usually $<10 \mathrm{~s}$ ), and they all succeeded not to blink for that extended period. However, in some cases, we had to manually hold the eye open to avoid interference from eyelashes. As the total measurement time was very short $(\sim 15 \mathrm{~s})$, the light intensity was low $(\sim 20 \mu \mathrm{W}$; many times lower than that in an ophthalmoscope used during regular eye check-up), and the light was incident on the white portion of the eye (i.e., not on the eyeball), the subject neither sensed the light nor reported any irritation or discomfort. Furthermore, the noninvasive and noncontact nature of our method eliminated the need for disinfecting the measuring probe. Immediately after the acquisition of spectral data, blood samples were collected from the subjects, and the complete blood count (CBC) was analyzed using an automated hematology analyzer (Sysmex KX-21). These data were later used for calibration and comparison.

In stage 1, the data of 302 (including 134 females and 48 children) participants were randomly selected for device calibration; the collected spectral response, as generated in the spectrograph and processed in the LabVIEW software, was stored in the tablet computer for further processing in terms of calibration.

In stage 2, the clinical assessment output of the calibrated device was compared with that of a standard hematology analyzer by using a statistically significant number of samples $(n=191)$. To demonstrate the repeatability and reproducibility of the proposed device, 56 and 50 patients in this stage were repetitively examined using our device by the same observer and by two independent observers, respectively.

\section{Results and Discussion}

The proposed device collects the spectroscopic signal of the blood from the human conjunctiva. We choose the conjunctiva of human eyes as the target organ to estimate the hemoglobin concentration as it is easily accessible, hosts well-oxygenated blood containing high-density vascular bed, ${ }^{25}$ and has high vascular visibility with a white background in all human subjects. In addition, the noncontact nature of our method ensures no change in tissue morphology, shape, or blood content in the target area. Although, the effects of low hemoglobin saturation on our measurements are unknown, the study by MacKenzie et al. ${ }^{25}$ showed that the blood in the bulbar conjunctival microvasculature is always highly saturated with oxygen. These advantages ensure that the proposed device can measure hemoglobin concentration with high accuracy and precision and without any interference from the other pathological conditions in subjects from all races. Figure 1(b) presents the spectral response of a normal volunteer and an anemic patient. There was an obvious difference in their absorbance spectra, clearly indicating the concentration of hemoglobin is much higher for the normal volunteer compared to the anemic patient.

\subsection{Calibration, Validation, and Statistical Analysis}

For device calibration, 302 patients (including 168 males, 48 of whom were children) of all age groups with hemoglobin levels ranging from 3.4 to $17.9 \mathrm{~g} / \mathrm{dL}$ were randomly selected for data collection; their information is listed in Table 1. The acquired data were then processed to enable quantitative comparisons with the blood hemoglobin levels obtained using the automated hematology analyzer. It has already been reported that the absorbance maxima of the blood at around $576 \mathrm{~nm}$ wavelength are directly related to the hemoglobin concentration present in the blood. ${ }^{23,24}$ Different characteristic wavelengths over the collected spectrum were selected for assessment, and the differential absorbance of light of wavelength 576 and $600 \mathrm{~nm}$ was found to be highly consistent with the blood hemoglobin levels of the patients. Figure 3 presents the dependency of the instrument index values (i.e., differential absorbance at 576 and $600 \mathrm{~nm}$ ) with blood hemoglobin levels. Clearly, there is a linear 
Table 1 Statistics of the patients' information.

\begin{tabular}{lcc} 
& For calibration & For validation \\
\hline Patients & $N=302$ & $N=191$ \\
Age $(\mathrm{yr})$ & $27 \pm 13$ & $26 \pm 12$ \\
& $(95 \% \mathrm{Cl}: 25 ;$ & $(95 \% \mathrm{Cl}: 25 ;$ \\
& $28, \mathrm{CV}: 49.15 \%)$ & $28, \mathrm{CV}: 49.15 \%)$ \\
Sex ratio M/F & $168 / 134$ & $108 / 83$ \\
Hb value $(\mathrm{g} / \mathrm{dL})$ & $10.15 \pm 3.43$ & $10.46 \pm 3.36$ \\
& $(95 \% \mathrm{Cl}: 9.76 ;$ & $(95 \% \mathrm{Cl}: 9.98 ;$ \\
& $10.53 ; \mathrm{CV}: 33.77 \%)$ & $10.94 ; \mathrm{CV}: 32.15 \%)$
\end{tabular}

Stage of anemia

\begin{tabular}{lll}
$\begin{array}{l}\text { Severely anemic } \\
\text { (3 to 7) }\end{array}$ & $N=63(20.8 \%)$ & $N=31(16.2 \%)$ \\
$\begin{array}{l}\text { Anemic (7 to 11) } \\
\text { Normal (11 to 18) }\end{array}$ & $N=120(39.7 \%)$ & $N=78(40.8 \%)$ \\
$\begin{array}{l}\text { Diagnosis } \\
\begin{array}{l}\text { Iron deficiency } \\
\text { anemia }\end{array}\end{array}$ & $N=18(59.4 \%)$ & $N=82(42.9 \%)$ \\
$\begin{array}{l}\text { Thalassemia } \\
\text { Leukemia }\end{array}$ & $N=17(5.6 \%)$ & $N=11(5.7 \%)$ \\
$\begin{array}{l}\text { Other anemia } \\
\text { (aplastic etc.) }\end{array}$ & $N=25(8.3 \%)$ & $N=14(7.3 \%)$ \\
$\begin{array}{l}\text { Other disorders } \\
\text { Not diagnosed } \\
\text { (screening) }\end{array}$ & $N=20(6.6 \%)$ & $N=15(7.9 \%)$ \\
$\begin{array}{l}\text { Normal volunteer } \\
\begin{array}{l}\text { Transfusion } \\
\text { (within 6 months) }\end{array}\end{array}$ & $N=114(37.7 \%)$ & $N=72(37.7 \%)$ \\
\hline
\end{tabular}

Note: $N$, sample size; $95 \% \mathrm{Cl}, 95 \%$ confidence interval of mean, $\mathrm{CV}$, coefficient of variation, and $\mathrm{Hb}$, hemoglobin concentration $(\mathrm{g} / \mathrm{dL})$.

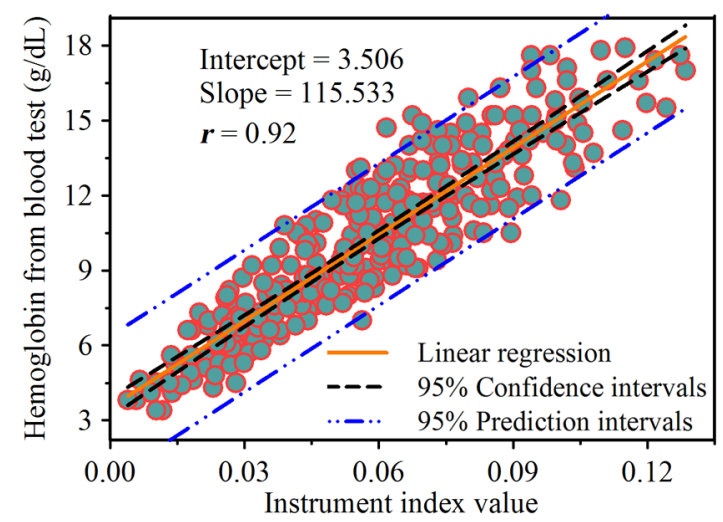

Fig. 3 Device calibration: linear regression of the hemoglobin level from the blood test and the instrument index value from our device $(r=0.92)$ with $95 \% \mathrm{Cl}$ and $95 \%$ prediction intervals. dependency between the instrument index values and blood hemoglobin levels. To calibrate the device and to determine the direct correlation with blood hemoglobin levels, the clinically measured hemoglobin levels (measured using a standard automated hematology analyzer) were plotted against the instrument index values. The linear regression curve with the $95 \%$ confidence intervals (CI) and $95 \%$ prediction intervals is shown in Fig. 3. The Pearson's correlation coefficient $r=0.92$ and the statistical parameters $P$ value $<0.0001$ and $F$ value $=1407.49$ indicate a strong correlation between the hemoglobin levels and instrument index values. The calibration equation obtained from the linear regression curve was $y_{i}=115.533 x_{i}+3.507$, where $y_{i}$ represents the hemoglobin level and $x_{i}$ represents the instrument index value. We used this equation in the LabVIEW software to estimate the hemoglobin level from the spectral information obtained using our device.

In the field of clinical measurement, a new measurement technique must often be compared with an established technique to evaluate whether the outputs of technique are sufficiently consistent for the new technique to replace the old one. Such investigations are often conducted through the Bland-Altman method. ${ }^{26}$ Hence, for the statistical significance of the proposed noncontact and noninvasive optical device for online assessment of the hemoglobin level, correlation and regression analyses were used following the reported literature. ${ }^{27-29}$ For assessing the agreement between the outputs of the conventional technique (automated hematology analyzer) and the proposed
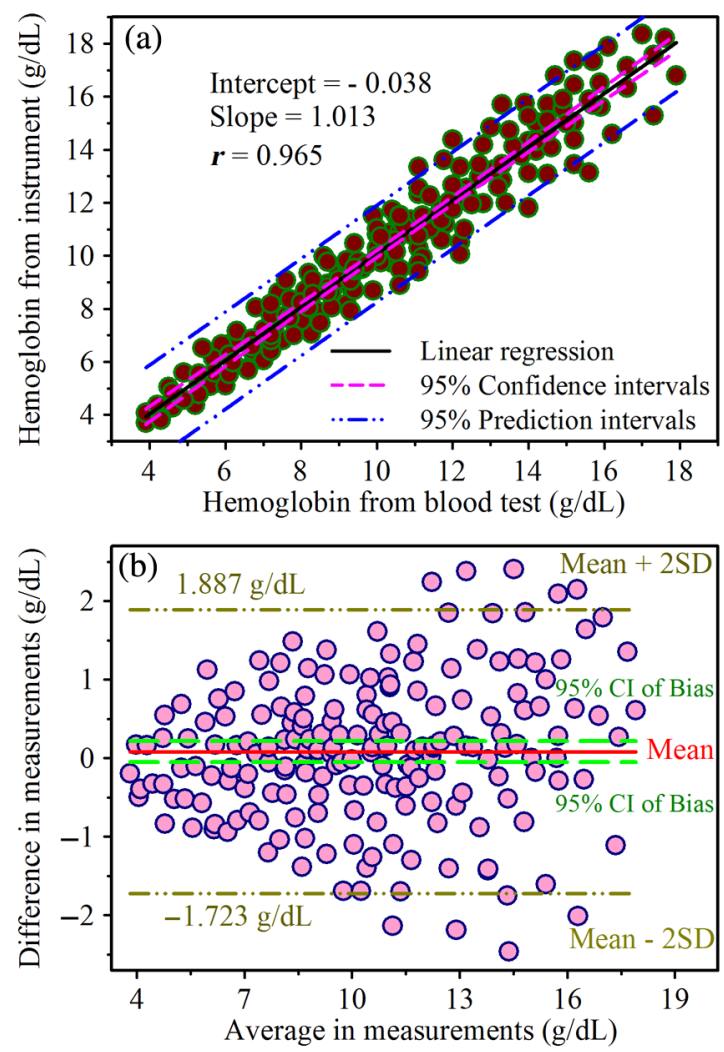

Fig. 4 Device validation: (a) linear regression of the hemoglobin level from blood test and the hemoglobin level from our device $(r=0.965)$ with $95 \% \mathrm{Cl}$ and $95 \%$ prediction intervals. (b) Bland-Altman analysis of the hemoglobin level from the blood test and that from our device with mean bias (continuous line), $95 \% \mathrm{Cl}$ of bias (dashed green lines), and $95 \%$ limits of agreement (bias \pm 2 SD, dot-dashed lines). 
optical device, the Bland-Altman method was used to determine the correlation coefficient. ${ }^{26}$

To compare the proposed point-of-care noninvasive hemoglobin detection method with an established conventional technique, a total of 191 patients (including 108 males, 26 of whom were children) of all age groups with hemoglobin levels ranging from 3.9 to $17.9 \mathrm{~g} / \mathrm{dL}$ were randomly selected. Their information is listed in Table 1. Figure 4(a) presents the linear regression analysis with the $95 \% \mathrm{CI}$ and $95 \%$ prediction intervals. The linear regression results (Pearson's correlation coefficient $r=$ $0.965, P$ value $<0.0001, F$ value $=2562.86$, slope $=1.013$, and intercept $=-0.038$ ) indicate a strong correlation between the two methods. For a more adequate comparison of the two methods, we applied the Bland-Altman method, which measures the agreement between two repeated measurements and the strength of the relationship between the measurements by using a plot of the difference between the methods against their average value [Fig. 4(b)]. ${ }^{30,31}$ A direct comparison of the two methods using this method yielded a bias of $0.082 \mathrm{~g} / \mathrm{dL}$ and SD of $0.921 ; 95 \% \mathrm{CI}$ for the bias was -0.049 to 0.214 and $95 \%$ limits of agreement [mean \pm 2 SD] were -1.723 to $1.887 \mathrm{~g} / \mathrm{dL}$ [Fig. 4(b)]; 95\% limits of agreement means that $95 \%$ of the differences are assumed to lie within these limits and how far apart the measurements obtained using the two methods are likely to be for most individuals. The accuracy of the proposed method relative to the reference method was calculated using the accuracy root mean square $\left(A_{\mathrm{RMS}}\right)$ as $\sqrt{ }\left([\text { mean bias }]^{2}+[\mathrm{SD}]^{2}\right) .{ }^{15}$ The obtained $A_{\mathrm{RMS}}$ of our method throughout the hemoglobin range was $0.924 \mathrm{~g} / \mathrm{dL}$, which indicates the high accuracy of the method. These results exhibit strong agreement between the conventional method and the proposed noninvasive as well as noncontact method of anemia detection.

Compared with laboratory reference values, the overall bias \pm the limits of agreement was $0.082 \pm 1.805 \mathrm{~g} / \mathrm{dL}$ for the proposed device, which is significantly lower than that of the commercially available noninvasive and invasive hemoglobin monitoring devices, such as Radical- $7^{\circledR}$ Pulse CO-Oximetry
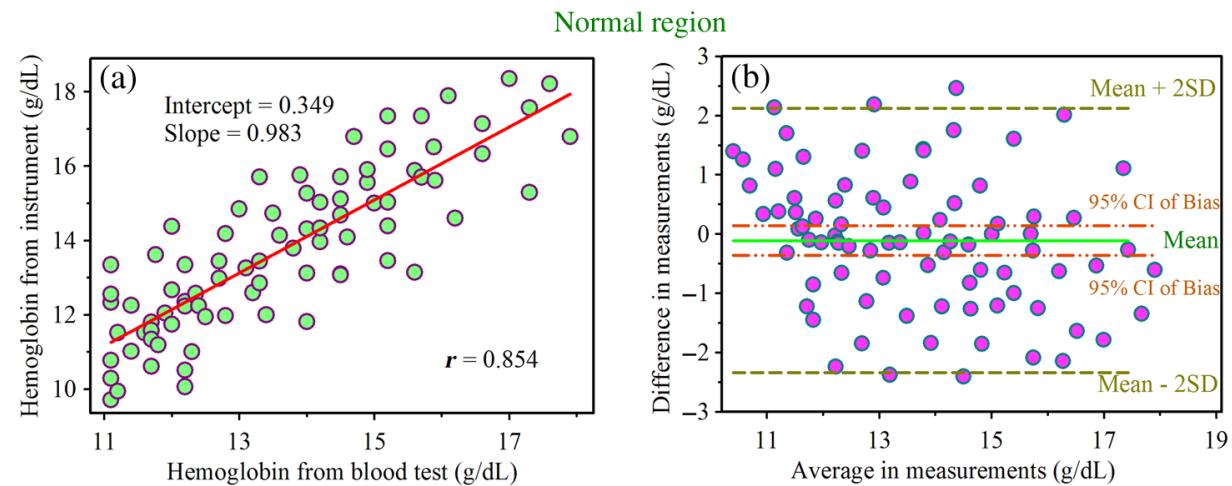

Mild anemic region
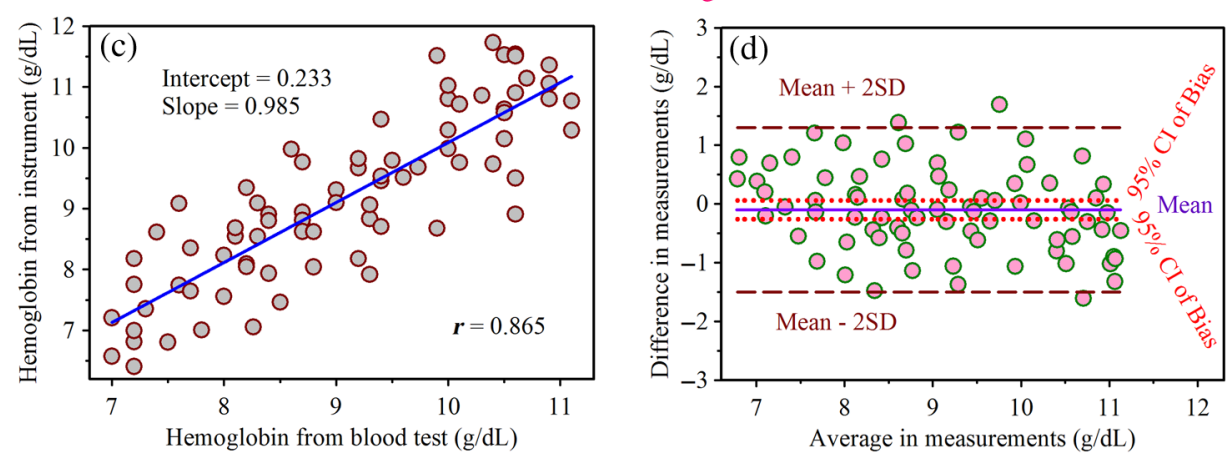

Severe anemic region
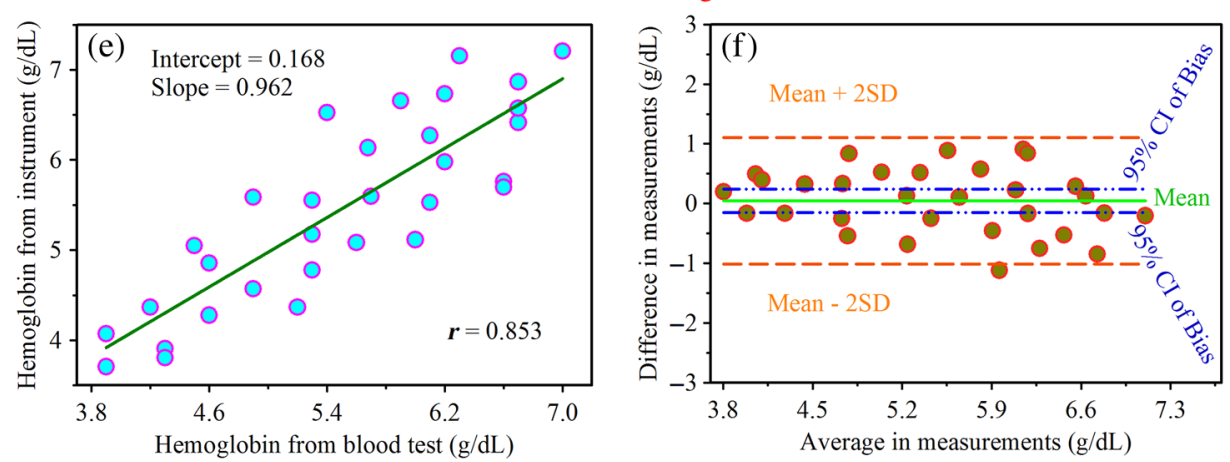

Fig. 5 Linear regression and Bland-Altman analysis of the hemoglobin level from the blood test and that from our device with mean bias (continuous line), $95 \% \mathrm{Cl}$ of bias, and $95 \%$ limits of agreement for (a) and (b) normal region, (c) and (d) mild anemic region, and (e) and (f) severe anemic region. 
$(-0.02 \pm 2.724 \mathrm{~g} / \mathrm{dL}), \mathrm{HemoCue}^{\circledR} 201+(-0.17 \pm 2.058 \mathrm{~g} / \mathrm{dL})$, HemoCue point-of-care device $(-0.4 \pm 2.6 \mathrm{~g} / \mathrm{dL})$, and hemoglobin monitoring using capillary blood $(-0.4 \pm 2.6 \mathrm{~g} / \mathrm{dL}) .^{15,16,32}$ Compared with laboratory reference values, noninvasive hemoglobin measurement with the proposed device has absolute and trending accuracy similar to those of widely used noninvasive and invasive methods of hemoglobin measurement. The proposed method is especially useful in low-resource settings, where conventional hematology analyzers are cost prohibitive.

Furthermore, the proposed device can efficiently classify anemia in the patient into the different stages defined by the WHO, the severe anemia, $<7 \mathrm{~g} / \mathrm{dL}$; mild anemia, 7 to $11 \mathrm{~g} / \mathrm{dL}$; and normal range above $11 \mathrm{~g} / \mathrm{dL}^{6,24}$ Therefore, for effective screening test, detection of hemoglobin level up to $11 \mathrm{~g} / \mathrm{dL}$ with high precision and accuracy is the main goal of our invention. According to the defined anemic range, we classified our results into three regions according to the aforementioned ranges [Fig. (5)]. Figure 5(a) presents the linear regression analysis for the samples in which the hemoglobin level exceeds $11 \mathrm{~g} / \mathrm{dL}(n=82, r=0.854, P$ value $<0.0001$, $F$ value $=198.10$, slope $=0.983$, and intercept $=0.349)$, and Fig. 5(b) depicts the Bland-Altman plot of two successive measurements made using the two different methods [mean = $-0.112 \mathrm{~g} / \mathrm{dL}, \mathrm{SD}=1.138$, and the calculated accuracy $\left(A_{\mathrm{RMS}}\right)$ is $=1.143 \mathrm{~g} / \mathrm{dL}]$. The correlation coefficient between the proposed device and laboratory-measured hemoglobin is the indication of good agreement between the two methods of measurement. The promising results imply that the device can detect and differentiate hemoglobin levels ranging from $11 \mathrm{~g} / \mathrm{dL}$ onward with high accuracy.

On the other hand, the $\mathrm{CBC}$ results revealed that the hemoglobin levels were 7 to $11 \mathrm{~g} / \mathrm{dL}$ in 78 samples; Fig. 5(c) presents the corresponding linear regression, which confirms that the clinical hemoglobin level measured using the automated hematology analyzer correlates strongly with the hemoglobin level measured using our noncontact device $(r=0.865$, $P$ value $<0.0001, \quad F$ value $=206.12, \quad$ slope $=0.985, \quad$ and intercept $=0.233$ ). In addition, the Bland-Altman method yielded a bias of $-0.099 \mathrm{~g} / \mathrm{dL}$ and SD of 0.713 ; the $95 \% \mathrm{CI}$ for the bias was -0.260 to 0.061 and $95 \%$ limits of agreement were -1.498 to $1.298 \mathrm{~g} / \mathrm{dL}$ [Fig. 5(d)]. The high accuracy of the device $\left(A_{\mathrm{RMS}}=0.719 \mathrm{~g} / \mathrm{dL}\right)$ in this region highlights the strong potential of the device. These results indicate that the output of the noncontact anemia detection device is highly consistent with that of the conventional method. The $95 \%$ limits of agreement suggest that the device is relatively more sensitive in the anemic region.

Finally, to establish the performance of the device in the severe anemia region, 31 samples were analyzed. Figures 5(e) and 5(f) present the linear regression analysis $(r=0.853$, $P$ value $<0.0001, F$ value $=77.25$, slope $=0.962$, and intercept $=$ 0.168 ) and the Bland-Altman plot (bias $0.043 \mathrm{~g} / \mathrm{dL}$, SD 0.542, $95 \%$ CI for the bias -0.154 to 0.241 , and $95 \%$ limits of agreement -1.014 to $1.101 \mathrm{~g} / \mathrm{dL}$ ), both of which reveal a strong correlation and agreement between the two methods. The accuracy of the device $\left(A_{\mathrm{RMS}}=0.54 \mathrm{~g} / \mathrm{dL}\right)$ in this region is highly comparable with commercially available devices. These promising results validate the ability of the device to detect severe anemia with extremely high accuracy. The Bland-Altman analysis confirmed that the proposed device is highly sensitive in the anemic region, more than that in the normal region, meaning that the device can accurately diagnose even the mildest forms of anemia as well as nonanemic participants' hemoglobin level and different degrees of polycythemia.

Finally, according to the CBC results, of the 191 samples with hemoglobin level in the 3.9- to $17.9-\mathrm{g} / \mathrm{dL}$ range, $109 \mathrm{had}$ anemia $(<11 \mathrm{~g} / \mathrm{dL})$; among the 109 anemic samples, 31 were severe anemia. The corresponding results obtained using our method are as follows: 112 patients were classified as anemic, among whom 35 were classified as having severe anemia. These results indicate that in the clinically most significant region (i.e., in the anemic region, $<11 \mathrm{~g} / \mathrm{dL}$ ), the performance of the proposed device is highly consistent with that of the current gold standard and that the device can determine anemia efficiently with extremely high accuracy. This device thus has the potential to provide CBC-quality measurement of total hemoglobin in point-of-care and austere medical environments, which do not have direct access to laboratory equipment. Moreover, the device is useful for diagnosing patients with any degree of anemia in low-resource settings in any region of the world.

\subsection{Repeatability Test}

To demonstrate repeatability, the hemoglobin levels of 56 patients were measured repetitively using the proposed device. We found excellent precision between two back-to-back measurements in the same subject by the same observer. The calculated SD and mean were almost the same in both measurements, with highly significant intraclass correlation (correlation coefficient $r=0.983, P$ value $<0.0001$ ). Linear regression analysis as shown in Fig. 6(a) further confirmed the accuracy of the
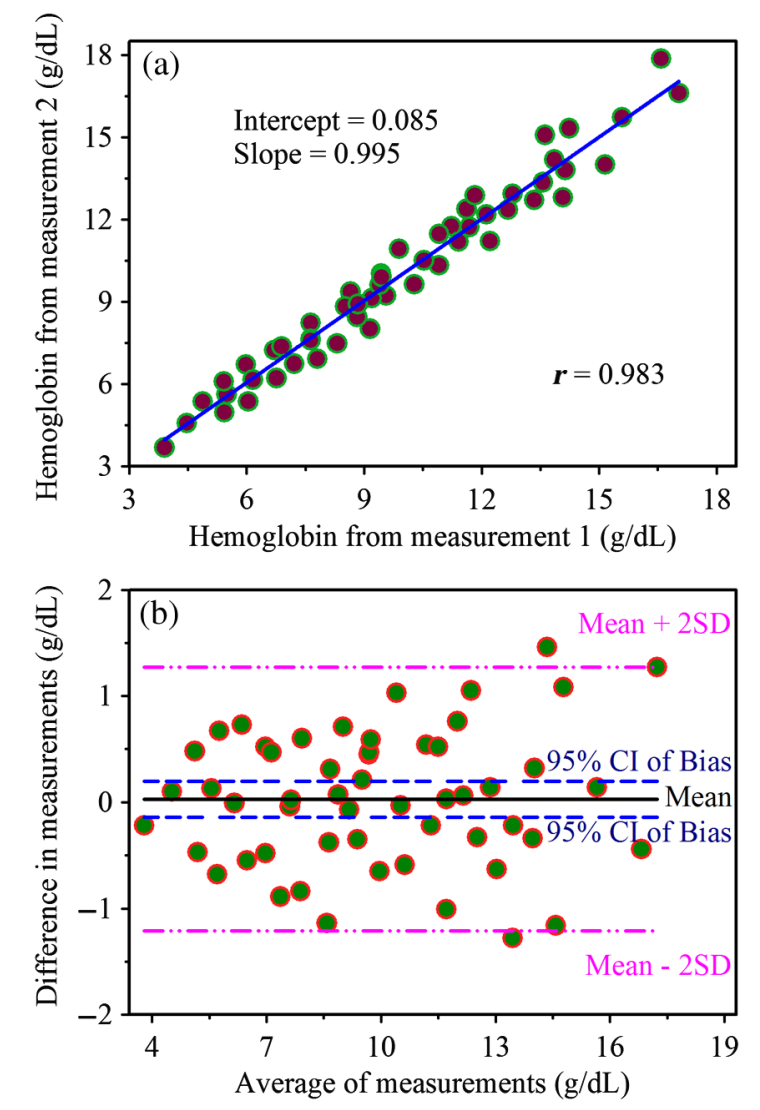

Fig. 6 Device repeatability: (a) linear regression analysis for two successive hemoglobin measurements in the same subject by the same observer and (b) the corresponding Bland-Altman analysis. 
two measurements $(F$ value $=1498.26$, slope $=0.994$, and intercept $=0.084)$. Figure $6(\mathrm{~b})$ presents the Bland-Altman plots of the measurements, wherein each point represents one patient (throughout the hemoglobin range, mean bias $=$ $0.03, \mathrm{SD}=0.633,95 \%$ limits of agreement $=-1.212$ to $1.272 \mathrm{~g} / \mathrm{dL}$, and $A_{\mathrm{RMS}}=0.634 \mathrm{~g} / \mathrm{dL}$ ). Ideally, the bias should be zero; the instrumental bias for our device was $0.03 \mathrm{~g} / \mathrm{dL}$, which is close to zero and exerts nearly no effect on the clinical hemoglobin measurement. These results suggest that the proposed noninvasive as well as noncontact device has excellent repeatability.

\subsection{Reproducibility Test}

Reproducibility of the device was assessed by measuring a clinically significant sample of 50 patients' hemoglobin levels two times in the same subject but by two independent observers. We found marvelous precision between two back-to-back measurements in the same subject by two independent observers. The measurements were highly precise, with nearly the same SD and mean values in both measurements and a significant correlation in the linear regression analysis (correlation coefficient $r=0.975, P$ value $<0.0001, F$ value $=993.68$, slope $=$ 0.982, and intercept $=0.134$ ) [Fig. 7(a)]. Moreover, the Bland-Altman analysis yielded a bias of $-0.052 \mathrm{~g} / \mathrm{dL}, \mathrm{SD}$ of $0.679,95 \%$ limits of agreement of -1.382 to $1.278 \mathrm{~g} / \mathrm{dL}$, and $A_{\mathrm{RMS}}=0.681 \mathrm{~g} / \mathrm{dL}$ [Fig. 7(b)]. The two measurements exhibited a nonsignificant bias and a close agreement. These
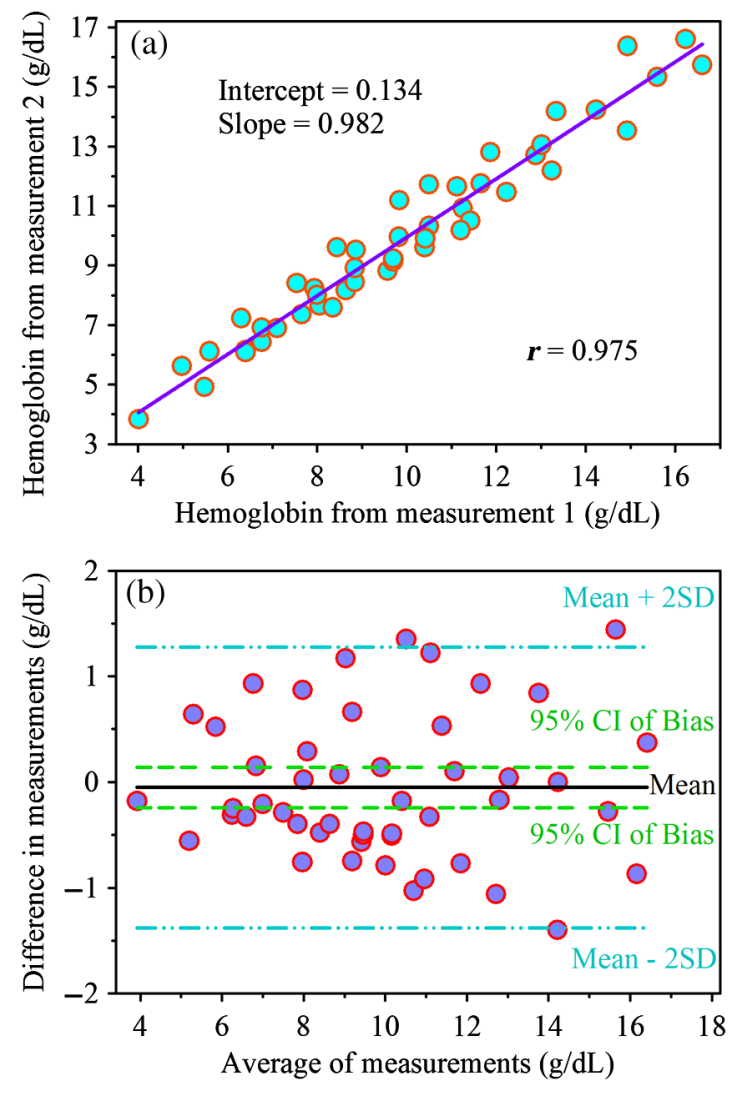

Fig. 7 Device reproducibility: (a) linear regression of the hemoglobin levels measured successively by two independent observers in the same subject by using the proposed device and (b) the corresponding Bland-Altman analysis. results indicate that the proposed noncontact device has high reproducibility.

\section{Conclusions}

We proposed a simple diffused reflection measurement technique for assessing hemoglobin levels and demonstrated that the vascular bed of bulbar conjunctiva is an ideal target area for this device. Through this spectroscopic method, which is invariable to skin color, age, and sex of the subject, we developed an inexpensive, noncontact, highly reliable, and efficient practical device for the online monitoring of hemoglobin levels in low-resource point-of-care settings. Moreover, the device can instantaneously transmit the generated report to a medical expert through e-mail, text messaging, or mobile apps. Furthermore, the device can classify the measured data into different degrees of anemia. In the clinically most significant regions [i.e., in the severe anemic region $(<7 \mathrm{~g} / \mathrm{dL})$ and mild anemic region $(7$ to $11 \mathrm{~g} / \mathrm{dL})$ ], the device has absolute and trending accuracy similar to those of widely used invasive methods of hemoglobin measurement. Moreover, this proposed noncontact, feasible, and inexpensive device for rapid anemia screening with extremely high precision and accuracy has high potential for further development and commercialization.

\section{Disclosures}

The authors have disclosed no conflicts of interest.

\section{Acknowledgments}

P.K.S. is thankful to the University Grants Commission (UGC, India) for providing the fellowship under the UGC-RGNF scheme. N.P. acknowledges the Department of Science and Technology (DST, India) for the Inspire Research Fellowship. The authors are thankful to the Indian Council of Medical Research for financial support (5/3/8/247/2014ITR), Department of Atomic Energy (India) for financial Grant 2013/37P/ 73/BRNS, and DST (India) for financial Grant SB/S1/PC$011 / 2013$.

\section{References}

1. Y. Balarajan et al., "Anaemia in low-income and middle-income countries," Lancet 378(9809), 2123-2135 (2012).

2. World Health Organization (WHO), "Micronutrient deficiencies," http:// www.who.int/nutrition/topics/ida/en/ (15 December 2016).

3. J. Punter-Villagrasa et al., "An instantaneous low-cost point-of-care anemia detection device," Sensors 15(2), 4564-4577 (2015).

4. M. M. Sirdah, A. Yaghi, and A. R. Yaghi, "Iron deficiency anemia among kindergarten children living in the marginalized areas of Gaza Strip, Palestine," Rev. Bras. Hematol. Hemoterapia 36(2), 132-138 (2014).

5. PATH, "Anemia detection in health services: guidelines for program managers," 1996, http://www.path.org/publications/detail.php?i=1201 (17 December 2016).

6. E. McLean et al., "Worldwide prevalence of anaemia, WHO vitamin and mineral nutrition information system, 1993-2005," Public Health Nutr. 12(4), 444-454 (2009).

7. WHO, Iron Deficiency Anaemia: Assessment, Prevention and Control: A Guide for Programme Managers, 2001, http://apps.who.int/iris/ bitstream/10665/66914/1/WHO_NHD_01.3.pdf?ua=1 (15 December 2016).

8. WHO, The Global Prevalence of Anaemia in 2011, 2015, http:// www.who.int/nutrition/publications/micronutrients/global_prevalence_ anaemia_2011/en/ (16 December 2016).

9. WHO, Global Nutrition Targets 2025: Anaemia Policy Brief, 2014, http://www.who.int/nutrition/publications/globaltargets2025_policybrief_ anaemia/en/ (16 December 2016). 
10. T. Srivastava et al., "Methods for hemoglobin estimation: a review of "what works'," J. Hematol. Transfus. 2(3), 1028 (2014).

11. L. D. Lilien et al., "Neonatal osteomyelitis of the calcaneus: complication of heel puncture," J. Pediatr. 88(3), 478-480 (1976).

12. R. Neville, "Evaluation of portable haemoglobinometer in general practice," Br. Med. J. 294(6582), 1263-1265 (1987).

13. J. Brown et al., "A hand-powered, portable, low-cost centrifuge for diagnosing anemia in low-resource settings," Am. J. Trop. Med. Hyg. 85(2), 327-332 (2011).

14. PATH, Noninvasive Anemia Screening, 2013, http://sites.path.org/ mnhtech/files/2013/04/FINAL_Anemia-Screening_23May2013.pdf (17 December 2016).

15. D. Frasca et al., "Accuracy of a continuous noninvasive hemoglobin monitor in intensive care unit patients," Crit. Care Med. 39(10), 2277-2282 (2011).

16. M. D. L. Lamhaut et al., "Comparison of the accuracy of noninvasive hemoglobin monitoring by spectrophotometry $(\mathrm{SpHb})$ and $\mathrm{HemoCue}^{\circledR}$ with automated laboratory hemoglobin measurement," Anesthesiology 115(3), 548-554 (2011)

17. J. W. McMurdy et al., "Diffuse reflectance spectra of the palpebral conjunctiva and its utility as a noninvasive indicator of total hemoglobin," J. Biomed. Opt. 11(1), 014019 (2006).

18. N. B. Hampson, E. D. Ecker, and K. L. Scott, "Use of a noninvasive pulse CO-oximeter to measure blood carboxyhemoglobin levels in bingo players," Respir. Care 51(7), 758-760 (2006).

19. A. Belardinelli et al., "Noninvasive methods for haemoglobin screening in prospective blood donors," Vox Sang. 105(2), 116-120 (2013).

20. T. Lister, P. A. Wright, and P. H. Chappell, "Optical properties of human skin,” J. Biomed. Opt. 17(9), 090901 (2012).

21. J. Ditzel and R. W. ST. Clair, "Clinical method of photographing the smaller blood vessels and the circulating blood in the bulbar conjunctiva of human subjects," Circulation 10(2), 277-281 (1954).

22. N. Polley et al., "Development and optimization of a noncontact optical device for online monitoring of jaundice in human subjects," J. Biomed. Opt. 20(6), 067001 (2015).

23. C. E. Carnicom, Morgellons: Altered Blood, 2011, http:// carnicominstitute.org/wp/morgellons-altered-blood/ (17 December 2016).

24. E. A. Tyburski et al., "Disposable platform provides visual and color-based point-of-care anemia self-testing," J. Clin. Invest. 124(10), 4387-4394.

25. L. E. MacKenzie et al., "In vivo oximetry of human bulbar conjunctival and episcleral microvasculature using snapshot multispectral imaging," Exp. Cell. Res. 149, 48-58 (2016).

26. J. M. Bland and D. Altman, "Statistical methods for assessing agreement between two methods of clinical measurement," Lancet 327(8476), 307-310 (1986).

27. J. M. Bland and D. G. Altman, "Calculating correlation coefficients with repeated observations: Part 2-correlation between subjects," Br. Med. J. 310(6980), 633 (1995).

28. M. J. Gardner and D. G. Altman, "Confidence intervals rather than P values: estimation rather than hypothesis testing," $\mathrm{Br}$. Med. J. 292(6522), 746-750 (1986).

29. J. M. Bland and D. G. Altman, "Statistics notes: correlation, regression, and repeated data," Br. Med. J. 308(6933), 896 (1994).

30. J. M. Bland and D. G. Altman, "Comparing methods of measurement: why plotting difference against standard method is misleading," Lancet 346(8982), 1085-1087 (1995).

31. J. M. Bland and D. G. Altman, "Applying the right statistics: analyses of measurement studies," Ultrasound Obstet. Gynecol. 22(1), 85-93 (2003).

32. G. Lindner and A. K. Exadaktylos, "How noninvasive haemoglobin measurement with pulse CO-Oximetry can change your practice: an expert review," Emerging Med. Int. 2013, 701529 (2013).
Probir Kumar Sarkar is currently working as a PhD research scholar under the supervision of Professor Samir Kumar Pal at S. N. Bose National Centre for Basic Sciences, Kolkata, India. He received his MSc degree in physics from the Indian Institute of Technology Kanpur, India, in 2013. The main focus of his work is on the spectroscopic studies of molecules and nanomaterials for potential applications in medical diagnosis and environmental pollution monitoring.

Sanchari Pal is pursuing her MBBS at Nil Ratan Sircar Medical College and Hospital, Kolkata, India. Her research interests include thalassemias, hemoglobinopathies, and hemopoietic stem cell transplantation.

Nabarun Polley received his BSc degree in physics and his master's degree in biomedical instrumentation from the University of Calcutta, India, in 2009 and 2011, respectively. Currently, he is pursuing his $\mathrm{PhD}$ under the supervision of Professor Samir Kumar Pal at $\mathrm{S}$. N. Bose National Centre for Basic Sciences, Kolkata, India. The main focus of his work is on developing and designing new biomedical tools using spectroscopic techniques.

Rajarshi Aich is currently a junior resident with the Department of Cardiology, Medical College and Hospital, Kolkata, India. He completed his MBBS in 2014 from Medical College and Hospital. His fields of interest are aberrations in posttranscriptional gene regulation mechanisms in tumor cells, particularly white cell malignancies.

Aniruddha Adhikari is currently working as a $\mathrm{PhD}$ research scholar under the supervision of Professor Samir Kumar Pal at S. N. Bose National Centre for Basic Sciences. His research interests include interactions of nanoparticles with living organisms, noncanonical DNA-protein interaction, structure-function relationship of ionotropic glutamate receptors, epigenetic regulation of embryonic stem cells, and telomere biology.

Animesh Halder received his MTech degree in bioelectronics from Tezpur University, India, and his BTech degree in applied electronics and instrumentation engineering from Haldia Institute of Technology, India. His interest includes the design and realization of instrumentation in biomedical studies and waveguide-based sensors.

Subhananda Chakrabarti is currently a professor in the Department of Electrical Engineering, Indian Institutes of Technology Bombay, Mumbai, India. His fields of interest include the growth and characterization of III-V compound semiconductor materials, optoelectronic devices, such as quantum dot photodetectors and solar cells, III-V device integration on germanium, and II-VI optoelectronic materials and devices. He has published more than 200 research papers in various international peer-reviewed journals, has three patents, and has authored three book chapters.

Prantar Chakrabarti is currently the departmental head of Haematology, NRS Medical College and Hospital, Kolkata, India. He completed his MBBS degree from Medical College and Hospital, Kolkata, in 1994 and his MD (general medicine) from the Institute of Post Graduate Medical Education and Research, Kolkata, India, and DM (clinical hematology) from the All India Institute of Medical Sciences, New Delhi. He has authored more than 35 papers and 16 book chapters.

Samir Kumar Pal is currently a professor in the Department of Chemical Biology and Macromolecular Sciences, S. N. Bose National Centre for Basic Sciences, Kolkata, India. His fields of interest include experimental biophysics in molecular recognition, bionano interface, biomedical instrumentation, and environmental pollution. He has authored more than 210 research papers in various international peer-reviewed journals, has 16 patents, and has authored five book chapters. 